\title{
Universal Science Part III
}

\section{S.D.S. Deshapriya}

\begin{abstract}
This paper is an outcome of a trial carried out to find a common methodology to address the different subjects together using circular thinking pattern which borrowed from Buddhism rather than addressing them separately. In addition to this, Buddhism which comprises of the perfect structure, immense innovative power and the broader ways of knowledge gaining are also utilized here to combine various subjects together. Due to the above fact, it further shows, how the subjects enhancements are feasible beyond prevailing knowledge level through patterns, simulations etc.

Universal Science(US) reveals that, all are at a transitional stage hence it has been the reason for every thing in the universe to have mutually opposite dual characters, representing the (concept of) unique origin (Satara maha buta) in one hand. An attempt has been made here to show the strength of US, by addressing the universal secrets such as the revolving of planets,gravitational forces, expansion of universe, occurance of common patterns in different things, repetitive nature of things such as rain circle, food circle etc. Further the new areas has also been introduced, such as the influence to the subjects by the common patterns at a higher level, need for exploring the whole area than one at a time, forming new theories using Buddhist texts, such as on universal conservation, as well as on new innovations etc. Hence, it is an occation of showing a link in spiritual \& material world in a different form.
\end{abstract}

(Part I \& Part II were published in June 2012 \& June 2013 journals respectively.)

\section{Origin \& the patterns}

It has been stated earlier that, according to Buddhism, every thing is formed by Satara Maha Buta (shortly say as satarabuta). Satarabuta are the unique characters and every thing in the universe is formed by satarabuta. Universal Science (US) also adhere to this as the satarabuta are with unique trend, unique properties \& uni-directional motive, while the World or the nature differs and it has scattered with a wider range of variety.

Therefore all different things in the world \& the nature are having a uniformity and uniqueness from the inception(birth). Hence they excel this hidden uniformity and uniqueness though they have differed very extensively in a wider range, by forming common (behavioural) patterns among different things. In other wards, this inherent \& hidden uniformity creates common(behavioural) patterns among different things though they are different. Hence the difference has grown always together with occurring the pattern among them while being It as the reason for occurring common patterns among different subjects. Accordingly, the secrecy of (the prevalance) persistence of common patterns, as we observed among different things is clearly revealed by US, through circular thinking in this manner which patronized by Buddhism. Occurrence of Similarities and the simulations among different subjects are the net effect of these common patterns. In other wards, the prevailing common patterns among subject, create the similarities in different things. Accordingly, the occurrence of simulations them selves as such, proves receprocatory the uniformity which existed as at the previous state of different things (and satara Butha? as the origin (inception) of every thing.)

It is the situation where the inherent uniformity(which existed from the inception) acts on the differentiation \& it Is due to the action of inherent hidden uniformity ,on variety that creats patterns. Hence these patterns are the repetition of common features \& qualities over \& over again among different things.

This will cover the pattern world entirely, which comprising of common patterns among various subject fields along the subjects axis.

Eng. S.D.S. Deshapriya, B.Sc. Eng (Moratuwa), C.Eng MIE(Sri Lanka) Senior Engineer, Road Development Authority. 


\section{Mutually two opposites:}

Generalizing the above issue of hidden uniformity in variety, it is a situation of an occurance of mutually two opposites together.

This shows, in contrast to the concept of Satarabhutha, which is unique, other things in nature are not unique, but they comprise of different qualities which are mutually opposite to each other. There fore, every thing is an assembly of mutually two opposite Qualities together which is acting simultaneously. Looking at a glance on world will be quite adequate to realize the above fact, in the light of comprising the world with mix up of things stiring continuously without flowing smoothly with a uninterrupted unidirectional trend \& the move. Hence it proves that the coexistence of (mutually) two opposites together, is being the common pattern and the phenomenon in nature. This phenomenon has been clearly stated in Buddhism by taking, as a example of mushroom with a bit of soil on top, to explain that the death arrises together with the birth \& the birth always accompanies death. Under the US, this pattern is taken as the fundamental theory of the universe \& based on which all other present scientific Theories are formed. This mutually two opposite theory (say TO theory) says that, "Coexistance of mutually two opposites is the phenomenon of the nature \& every thing is comprised of the qualities which are mutually opposite to each other."

This theory itself (which explains about the coexistence of (mutually) two opposites together) has been a paradox. Hence it can't be contained within the present binary logical frame \& there fore it is contained in the four quadrent logical frame(say 4QD NT ) (which will be explained later.)

US identifies that the above inherent paradoxity on which the nature has been based on, is responsible for the complexicity of nature \& the Universe. Hence, US is been able to apprehend the complexicity of nature in the four quodrent logical frame from its inception.

Accordingly, US explains that, entire world \& the nature is governed by this TO theory \&it is a universal pattern. While assuming the above fundamental theory of which based on four quadrant logic, US will be able to explain the secrets and phenomena in universe \& nature which cannot be explained in present science and through binary logic. Even though it is too early in Universal Science to comment about the universal secrets, same topic is taken here to show the versatility of this science. Nature prevails, hence it can't be disproved or denied. Hence it Is stressed here that, in any case of an error occurred in the process of developing US, it should go to the author since US is about the nature which can't be denied. As stated above, US identifies that compared to the concept of Satara buta which are with unique trend, unique properties \& uni-directional, nature comprises of mutually two opposite pairs, based on 4Qud rnt logic. Accordingly US is being able to give the most reasonable expression to the nature.

\section{Gravitation \& revolving}

In an another aspect, nature \& the world around us is changing \& moving very fast.

TO theory says that effect of hidden static feature is also in effect, simultaneously on this move. It is again an occurrence of two opposites pair together (as a result of unique static origin with the same trend).

Hence it has not allowed the nature to move freely but do form it to a circular \& rotational shape(formation). Hence the nature movements are circulated since it is the only shape (form) that can satisfy both move \& stay (static property)together simultaneously.

In other wards, it is the circular form, (as the only way) that has retained static feature with out hindering the fast movement of the nature;hence being a mediating technique. With time same rotational movement will continue over \& over again in the time axis. Hence it also is showing the repetitive pattern of nature. Rain circle, food circle, climetic patterns are some examples to the above circular behavior.

As in a different example, Planets are at far a part. Due to the Effect of TO theory, is reacting on it to oppose this scattered nature as a hidden inherent feature.This hidden feature works as to take them closer again to the original state of uniqueness, resulting to form gravitational force (" $\mathrm{g}$ ") which is the attraction in the universe.In the other hand," $g$ " acts as the only way to take planets closer, with out hindering them to be at far;hence being a mediating tool. When " $\mathrm{g}$ " had been occurred, it had never been ended, but is being continued right 
through out. Since "g" is been continued with time, uninturpted manner, is again a repetitive pattern of nature in the time scale. (Further explanation on " $\mathrm{g}$ " is given later.)

Summerizing the above three examples, of (1)Uniformity in variety (2)stay property in move \& (3) being closer against being at far a part, were discussed here.

Even these examples of different types, which were randomly selected has shown the effect of coexistence of two opposites to the nature.

Hence the coexistence of two opposites in nature, as revealed by US, has made the nature itself to be a paradox which operates on 4QD NT. Logic, (as explained earlier.)More than that, these examples have shown that, this coexistence of mutually two opposites has made the whole nature, also to be an ever repetitive, as a remarkable feature( another common inherent phenomenen ), in contrast to satarabuta (concept) which is having unique property with a uni directional move \& the motive. In short, what we experience as the fast changing nature,as the world pattern, is merely a rotation in nature.

Accordingly, US explains mainly two types of rotations (repetitions), both in subjects axis (as patterns) \& in the time axis, covering the entire repetitive nature of Universe, which is governed by the two opposites Theory. Hence, all type of repetitions \& the cyclic actions are totally contained into US through this Theory .

In the circumstances, rotational movement of the earth etc. can be taken up. As stated above, repetition is the nature of universe both in time axis \& in subjects axis (among subjects). It extends its material form as (physical) rotation. In other wards, rotation is non other than the physical form of repetition itself. Hence the earth is also rotating (revolving) in accordance with the repetitive nature of universe which govern by the TO Theory. Further, similarly all the planets are also revolving as a repetitive behavior while fulfilling the above theory. Hence the revolving of planets is not a separate \& an isolate incident any more, but has to be treated as the physical form of the repetitive nature of the universe. These are the ordinary circles acting at the basic level.

(Rotation of earth around the sun etc. is taken later).
There are some important points, immerged in this stage. US reveals that in general, some governing theories at a higher level,are effective to many subjects (beyond one subject limit).This explores a new dimension to the present science. Hence some incidence can not be explained or contained with in one subject frame since they act according to a common pattern at a higher level, which Is being effective to many subjects \& accordingly which the subject under consideration also acts.(Further explanation on this is given later.)

\section{Gravitation further}

Prevaling common patterns among various subjects are responsible for forming simulations \& the similarities in respective subject field. (pl. see the $1^{\text {st }}$ para.) It Is also being used in the world as a common methodology for new innovations as explained in part 1 of this series.

Further these simulations are very important to explore the previous states as well as the secrecy of the Universe and hints that, the whole universe has occurred from one unique thing (ie. Satara Butha) as it's previous state. According to the scientists, origin is due to the Big Bang and this concept is not contradicted but (partially) supported by the revealing of Satara Bhuta, which is being in line with as one source of origin. How ever compared to the Big Bang, Satara Bhuta concept is far fundamental and profounding, since it comprises of four common qualities features \& patterns with out having a sence \& signs in time(scale).

Accordingly, as a new openion, the occurrance of Gravitational forces is also explained again through circular (pattern) thinking \& simulations. As stated above, different non physical things even though they are different, show their (residual)connection or the affinity to their original state of uniformity and the uniqueness (of Sathara Bhuta), as its property, by forming common patterns (\& the simulations as the out come). Similarly, whole Universe which is comprising of unlimited number of planets even though they are far a part are showing physically their connection, still remaining) to each other as well as the affinity and the tendency to become the previous unique state (to collect together )by forming gravitational forces. (attraction to combine each other \& to become unique). According to the circular thinking, it shows that the physical as well as non-physical 
things follow the similar pattern of tendency \& to become the previous state or showing the connectivity(residual) to the previous state (of Satara Bhuta of common unique origin) as a property \& a governing rule.

Hence the reason for gravitational forces could be summeried here as due to the common governing pattern \& the property of (residual) affinity \& the connectivity which remains still, to its previous state of uniqueness(satara bhuta) or as the physical form of common patterns. (ie. similar to the common patterns prevailing among subjects).

Accordingly the pattern thinking shows in the first instance that the gravitational forces are a universal occurance \& not limited to this globe only, as what Sir.I Newton found earlier.

\section{Future exploration}

In this instance, US suggests four important points as a guide for future exploration process in any subject field.

1. Due to the failure in single exercises targeting one answer at a time separately, US direct for exploring the whole range (area)since all are interconnected.

Hence searching the reason for " $\mathrm{g}$ " through this planet alone is futile, since it is merely a effect of causes of which common \& connected to the whole universe as well to its origin. Hence the area of test field should be the whole universe for the above exploration.

2. Since the present science has almost reached its margin, solutions will have to be sought, here onwards, from out of frame, through pattern thinking to avoid present delay \& the stagnation in all subject fields.

3. since all subjects tends to connect at the next higher level, hence a Common(behavioral)pattern itself, being at the higher level, will be the answer/reason/nature/solution (pattern solution) \& revealing of a secret or a phynomina, which are being searched for now \& accordingly which showing their indevidual behaviour so. Simply, the common (behavioural) pattern, as above, will be the nature which can not be questioned or reason out further.This has to be identified as the break even point of present science which is working at the individual subject level. Hence it is the reason for the present science to resort to US to search for the governing theories common to many subjects, which are working at a higher level. Accordingly, " $g$ " in this case has occured by obeying to the common governing pattern of affinity to its previous (state of)uniqueness ". Further these pattern solutions will act as the boundry \&the guide for any further study there onwards.

4. More success will be in hypothetical, empirical \& the pattern approach over the typical analytical approach since the analysis will be impractical \& it will have to go beyond the subject boundries.

According to the Buddhism, origin of universe or the origen of life can't be found and only the cause of persistence of life is identified as 'tanha'. Hence according to the pattern thinking cause of occurrence and the cause of persistence are not the same but the two different causes. a. Cause of occurrence $b$. cause of persistence.

Hence the cause of persistence has no relation to its origin.In this context it is not correct to deside the origen of universe based on persistence by (towards backward) extrapolating etc. Hence US questions the openions now suggests by the scientists about the exploration of universe, which based on the persistence. Hence it makes the room for a hypothetical approach, as more reasonable way to explore the universe.

\section{Creations \& conservation}

According to the Buddhism, new born is neither a previous creature nor the not so.This would give a good opportunity to ascertain the logical frame which the Buddhism is based on. Both sides of the logic Is denied here in the forth quadrant. Hence this logical frame is more suitable to cover the incidents or the situations, where the binary logic fails. Specially the paradox situations could be well contained only in this logical frame. This is comprised of four number of quadrants which covers all the situations. Hence, this is called as Four-Quadrent logical frame.

US which is patronized by Buddhism, also formed on the four quadrant logical base. Being privileged so, will give the opportunity 
for US to synchronize with nature since the natute itself acts on this four quadrant base. One day present science also will have to come to the threshold of this logic, if it also has to apprehend the nature very correctly .

As explained above, the incidence of new born is also based on the four quadrent logic.

In other aspect, above phenomenon is not limited to the incidence of new born but it hints; hence US reveals that it as the common pattern of the conservation nature of the universe. Hence it is revealed that the incidence of new born also has followed the same Universal pattern which can be generalized as Theory of Universal Conservation.

\section{Theory of universal conservation}

"Every thing in the universe is preserved Nothing can be created newly nor destroyed. Hence neither additions nor the deductions are occurred in the Universe. Changers or new things which occurring in the universe are merely the repatitions or the transformations among the things including mass \&the Energy."

Hence two theories of mass \& energy conservation, which appears in the present science and the above new innovation Theory as explained under US are merely the each indevidual versions with respect to the each category of mass, energy \& new innovations separately.

Accordingly, in addition to the Theory of conservation, new theory for innovation also can be derived .

"Nothing can be created nor destroyed. Hence no new things (innovations) can be created. New innovations are the similarities or merely the different forms of the previous stage. New innovations in one subject field is just a transformation of one subject similarity to another subject through common patterns prevailing among the different subjects".

This is not to discourage or under value new innovations but to make them more closer \& familier and to take the new innovations to the arms length.

Methodologies for new innovations are in two types as revealed by Buddhim in $1{ }^{\text {st }}$ part of this series. Cause \& effect and the pattern \& simulations are the two types there in. In order to satisfy the above theory which says that, there is no new creations, it reveals that "cause
\& effect" method also should follow a connectivity or a pattern. However causes \& the effect are apperently far different. In this context, when realizing that the effect is merely the different version of (prior) causes \& only as the transformation, it will satisfy the above theory (of no new creations, etc.). Accordingly, both type of methods are brought more closer to promote new innovations. (All are merely the effects what we observe in the nature. All these effects are occurred due to the causes \& nothing is occurred without a cause.)

\section{Behaviour of universe/simple harmonic motion}

Above dynamic \& static opposite pair can also be called as Change \& the constant for easy reference, and the repetitive nature is experienced right through out as the universal pattern, in accordance with two opposites theory as explained earlier. All are living in an ever changing universe, dominated by $\mathrm{TO}$ Theory of which based on $4 \mathrm{Qu}{ }^{\text {nt. Logic. Hence }}$ while changing, it is maintaining the constant feature also, as obeying to the above theory.But the cruciel problem here Is, how to keep on changing the universe while being constant ? Hence the repeated expanding \& contracting would have to be the only behavioral pattern that the universe can under go with time to satisfy above theory(being the volumetric average as constant). Simply it is the simple harmonic motion that follows. When trying to expand, is not allowed due to the property of affinity to the prior uniqueness, while obeying to the TO theory; hence starts contracting again by completing a one circle. In the time axis, same circle is repeated over \& over again. This behavioural pattern of expansion \& contraction has also been explained in Agganna discourse as "avatta- sanvatta \& vivatta".

When summerizing the universal behaviour, as its" volumetric form", while universe is following the receprocatory \& the simple harmonic pattern, it also follows the revolving pattern as its" motion form" as explained in $\underline{5 \text { th }}$ para of gravitation \& revolving. While engaging in both type of repetitive motions, in accordance with To theory, simple harmonic pattern is becoming again non other than, an another form of circular pattern itself, due to the fact that the liner projection of circular motion on its diagonal is being a simple harmonic one.

In a situation where the expansion \& the contraction, is clearily reveald through US, as 
the univeral pattern while securing its repetitive nature obeying to the To theory, scientists opines the universe as ever enlarging. Hence through US is compelled to question them for their stand \& the reasons behind.

\section{Conclusion}

Difference in nature is arrested by US through the hidden common patterns there in. Accordingly, a common theory have been formed about the coexistence of mutually two opposites. Reason for occurring a paradox as such, as revealed by the US is due to the unique origin which excels the original features even at latter stages. Hence US suggests that, gravitation which experiences is also a direct effect of it. Some suggestions also have been forwarded for future exploration by considering the interconnectivity nature of things since the analytical approach for each individual goal is impractical \& not successful. It has been proposed here for governing theories at higher levels, as a new concept, which are effective \& common to many subject fields. Above mensioned two opposites are responsible for the repetitive nature of the world \& the rotation as the physical form of it.

This will sum up with convincing all that, every thing in universe is non other than, about the story of circles(chakra) in various forms,(both in physical \& non-physical state), such as cycles (repatitions), patterns, revolving, simple harmonic motion, continuing etc.

\section{References}

1. News Paper articles including "Mihindu Himiyange Amba Prashnaya" of Professor Nalinda Silva.

2. News Paper articles of Buddhist Scholar, Professor Hettiarachchi etc. 